\title{
Large Right Ventricular Fibroma in a 6-Month-Old Infant
}

\author{
Alice Horovitz • Irene E. van Geldorp • \\ François Roubertie $\cdot$ Jean-Benoit Thambo
}

Received: 4 April 2012/Accepted: 9 May 2012/Published online: 29 May 2012

(C) The Author(s) 2012. This article is published with open access at Springerlink.com

\begin{abstract}
This report describes the case of a 6-month-old girl with a large cardiac fibroma in the right ventricle. Ventricular tachycardia associated with the fibroma was successfully treated with amiodarone. At the age of 3 years, surgical resection was indicated because of right ventricular outflow tract obstruction caused by progression of the tumor. The fibroma was successfully resected, and further follow-up evaluation was uneventful.
\end{abstract}

Keywords Cardiac tumor $\cdot$ Fibroma $\cdot$ Ventricular tachycardia

Primary cardiac tumors in children are rare. The incidence is reported to be $0.03-0.32 \%$, with fibroma accounting for approximately $25 \%$ [3]. Fibromas, usually single and large, are most commonly found in the left ventricular free wall or septum and less commonly involve the right

\footnotetext{
A. Horovitz · I. E. van Geldorp ( $₫)$ · J.-B. Thambo

Department of Congenital Heart Disease,

Bordeaux University Hospital, 1,

Avenue de Magellan, 33600 Pessac,

France

e-mail: i.vangeldorp@maastrichtuniversity.nl

I. E. van Geldorp

Cardiovascular Research Institute Maastricht,

Maastricht University, Maastricht,

The Netherlands

F. Roubertie

Department of Cardiothoracic Surgery,

Bordeaux University Hospital, Pessac,

France
}

ventricle (RV) or atria. We report a case of a young girl with a large cardiac fibroma in an atypical location.

\section{Case Report}

In a 6-month-old girl, a large cardiac tumor in the RV was discovered during evaluation for a soft systolic murmur. Except for the soft systolic heart murmur, the overall pediatric examination was normal. Echocardiography showed a large $(4.0 \times 4.5 \times 4.0 \mathrm{~cm})$ homogeneous mass appended to the RV free wall (Fig. 1). There was neither significant RV outflow tract obstruction nor stenosis of the tricuspid valve. Cardiac computed tomography (CT) and magnetic resonance imaging (MRI) confirmed the presence of a large intraventricular solid mass developed at the expense of the RV lateral wall (Fig. 2). Short-term follow-up assessment was complicated by an episode of symptomatic ventricular tachycardia, which was successfully treated with electric cardioversion and amiodarone. Oral therapy with amiodarone was continued under close echocardiographic and rhythmic monitoring with uneventful follow-up evaluation for 2 years.

At the age of 3 years, surgery was clinically indicated because tumor progression had resulted in obstruction of the RV outflow tract. The tumor was completely resected, and the resection margins were plicated (Fig. 3). Histologic examination of an intraoperative tumor biopsy showed abundant proliferation of fibroblasts with collagen deposition, which confirmed the diagnosis of cardiac fibroma.

The girl's postoperative course was uneventful. Because arrhythmia did not occur during a 6-month follow-up period, the amiodarone treatment was discontinued. Long-term follow-up evaluation was without complications and showed normal cardiac function without recurrence of the tumor. 


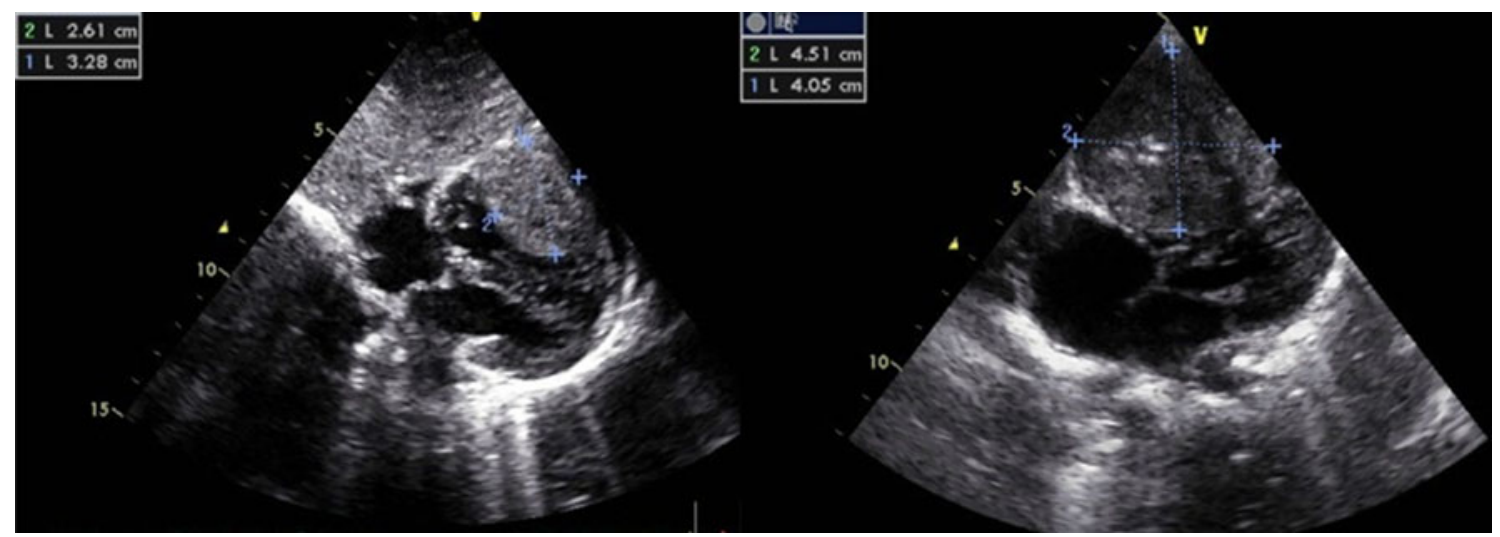

Fig. 1 Transthoracic echocardiography showing a large homogeneous mass originating from the right ventricular free wall

Fig. 2 The presence of a solid tumor confirmed by magnetic resonance imaging
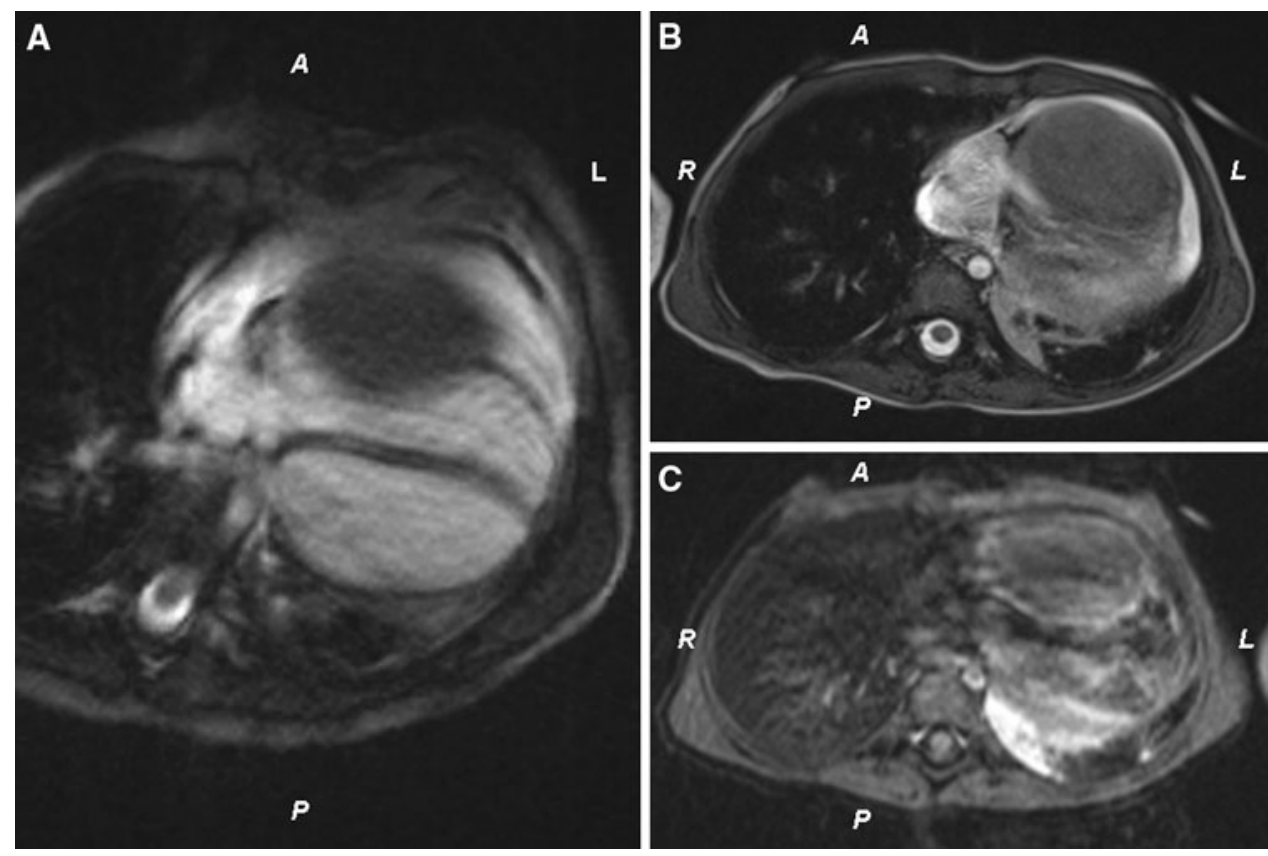

\section{Discussion}

Although fibromas are histologically benign, they can induce serious cardiovascular complications, including intracavitary obstruction and rhythm disturbances, as illustrated by the reported case and described in the literature [3]. Although treatment of symptomatic fibromas consists of complete excision [4], the management of asymptomatic fibromas remains controversial. Surgery may be complicated by tumor location and size but is reported to result in extended symptom-free survival with acceptable low operative morbidity and mortality, even for patients with large fibromas [1].
Fibromas are not likely to decrease [2], and because they often are associated with clinically significant and potentially life-threatening arrhythmias [3], we think patients should be considered for early surgical treatment when cardiac tumors, probably fibromas, are diagnosed. However, a more pragmatic strategy may be preferred in cases of uncertainty concerning the nature of the tumor or its cleavage plane, when excision of the tumor might affect the integrity of the ventricle, or when individual contraindications for surgery exist. In the reported case, surgery was postponed by treatment of ventricular tachycardia and careful monitoring. For small children, the latter strategy may increase the chances for successful and uncomplicated surgery. 

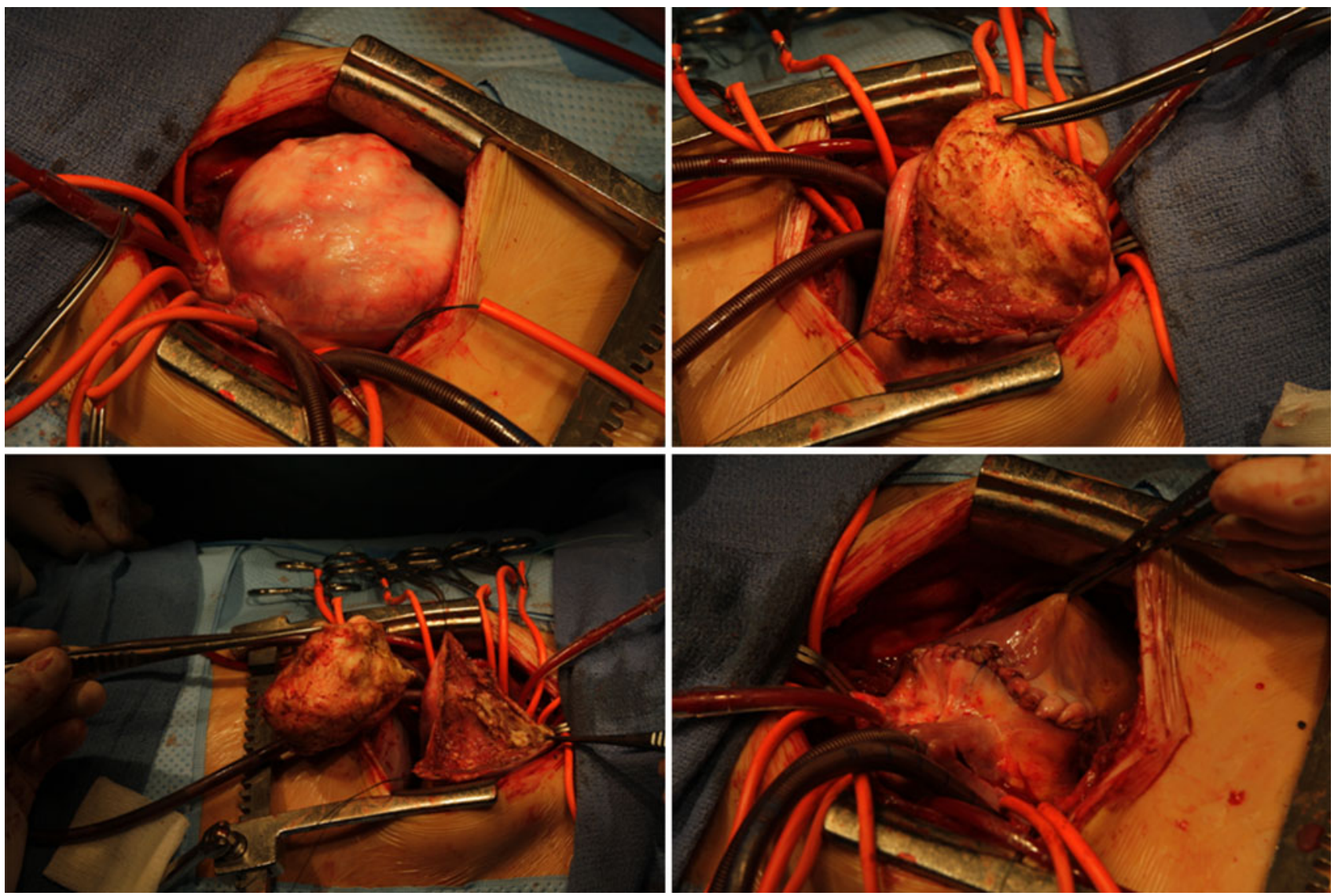

Fig. 3 Macroscopy of the encapsulated tumor during the different stages of surgical resection and at its total resection (lower right panel)

Acknowledgment Irene E. van Geldorp is financially supported by the Dutch Heart Foundation (Grant NHS-2010T078).

Open Access This article is distributed under the terms of the Creative Commons Attribution License which permits any use, distribution, and reproduction in any medium, provided the original author(s) and the source are credited.

\section{References}

1. Cho JM, Danielson GK, Puga FJ, Dearani JA, McGregor CG, Tazelaar HD, Hagler DJ (2003) Surgical resection of ventricular cardiac fibromas: early and late results. Ann Thorac Surg 76:1929-1934

2. Freedom RM, Lee KJ, MacDonald C, Taylor G (2000) Selected aspects of cardiac tumors in infancy and childhood. Pediatr Cardiol 21:299-316. doi:10.1007/s002460010070

3. Miyake CY, Del Nido PJ, Alexander ME, Cecchin F, Berul CI, Triedman JK, Geva T, Walsh EP (2011) Cardiac tumors and associated arrhythmias in pediatric patients, with observations on surgical therapy for ventricular tachycardia. J Am Coll Cardiol 58:1903-1909. doi:10.1016/j.jacc.2011.08.005

4. Takach TJ, Reul GJ, Ott DA, Cooley DA (1996) Primary cardiac tumors in infants and children: immediate and long-term operative results. Ann Thorac Surg 62:559-564 